\title{
Servicio social e iglesia católica: Condicionantes contextuales de su relación en los primeros años de la Escuela de Servicio Social de la Universidad de Costa Rica
}

\section{Social service and the catholic church: Contextual constrains of its relation in the first years of the Social Service School of the University of Costa Rica}

Milton Ariel Brenes Rodríguez ${ }^{1}$

\begin{abstract}
Resumen
El artículo pretende aportar al esclarecimiento de la discusión sobre la influencia de la Iglesia Católica en los primeros años de la Escuela de Servicio Social de la Universidad de Costa Rica, los cuales se sitúan en la coyuntura de la década de 1940. El documento fue elaborado a partir de la consulta de referencias bibliográficas y fuentes primarias de archivos de la época en estudio. El análisis concluye que la Iglesia Católica pudo considerar a la Escuela de Servicio Social de la Universidad de Costa Rica como un eventual escenario para la reproducción de su proyecto eclesiástico, pero las condiciones históricas a nivel internacional y nacional impidieron la reproducción formal de este proyecto.

Palabras claves: Servicio Social, enseñanza superior, iglesia católica, Universidad de Costa Rica Escuela de Servicio Social
\end{abstract}

\begin{abstract}
The article intends to provide the clarification of the discussion about the influence of the Catholic Church in the first years of the Social Service School in the University of Costa Rica, wich are situated on the juncture of the fortys. The document was created from the consult of bibliographical references and primary sources of files from the time on study. The analysis concludes that the Catholic Church could consider the Social Service School of the University of Costa Rica as an eventual scene for the reproduction of its ecclesiastical project, but the historical conditions at international and nacional level prevent the formal reproduction of this project.

KeyWords: Social Service, higher education, catholic church, University of Costa Rica, Social; Social Service School
\end{abstract}

1. Universidad de Costa Rica, Escuela de Trabajo Social, milton.brenesrodriguez@ucr.ac.cr

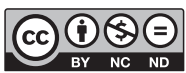




\section{Introducción}

En el presente escrito se aporta un estudio sobre la influencia de la Iglesia Católica en la Escuela de Servicio Social ${ }^{1}$ de la Universidad de Costa Rica. El análisis de dicha relación se entabla a partir de los condicionantes históricos nacionales e internacionales que posibilitan la existencia de la profesión, y la demanda de un respectivo espacio formativo.

El documento tiene como antecedente la investigación realizada por el autor de este escrito en el Trabajo Final de Graduación de la Licenciatura en Trabajo Social de la Escuela de Trabajo Social de la Universidad de Costa Rica, el cual aporta especialmente en el último apartado del texto. La revisión bibliográfica y la consulta de fuentes primarias fueron los principales insumos empleados para la construcción de este artículo.

\section{Génesis del Servicio Social en Occidente}

Las condiciones que propiciaron el surgimiento del Servicio Social se encuentran inicialmente situadas en Europa Occidental y en la costa Este de los Estados Unidos, durante el último cuarto del siglo XIX. En dicho contexto, las economías europeas y estadounidenses fueron más amplias en sus bases geográficas, así como en sus modos de operar. Hobsbawm (2017) afirma que el Reino Unido poseía para 1914 la centralidad de la económica occidental, seguido por Francia, Alemania, Estados Unidos, Bélgica, los Países Bajos y Suiza; en lo que respecta a los avances impulsados por las revoluciones industriales, se pudo evidenciar ampliaciones en las comunicaciones y las tecnologías; los aspectos en mención, propiciaron dinámicas de acumulación que favorecieron el establecimiento del capitalismo.

El autor en mención señala que las industrias de tales economías se caracterizaron por la producción de textiles, carbón, hierro, acero, armamento, construcción de barcos y químicos, siendo los sectores trabajadores los encargados a nivel operario de realizar estas labores como medio de vida (Hobsbawm, 2017). Ante tales situaciones, las masas trabajadoras, principalmente, en Europa se organizaron mediante agrupaciones y partidos políticos exigiendo mejoras en las condiciones de vida imperantes. La base ideológica empleada por una gran parte de estos grupos fue el socialismo revolucionario, conduciendo a un confrontamiento con la dominación burguesa de la época (Hobsbawm, 2017).

En estas regiones es posible identificar el ascenso de la pauperización de los grupos trabajadores, conjugado con una serie de acciones de los estados nacionales, quienes prestaron atención a las demandas de los sectores trabajadores, movidos por el interés de preservar el orden capitalista en asentamiento para la época. Dichas acciones son traducidas en asistencia social, siendo posible identificar su inicial implementación en Europa, pero más específicamente en Inglaterra a partir de la segunda mitad de siglo XIX.

Al ser Inglaterra cuna de la revolución industrial y, por consiguiente, del movimiento trabajador en Europa, como experiencia del Estado Nación posee circunstancias específicas para buscar tempranas respuesta a la naciente cuestión social; Martinelli (1989) quien desarrolla este planteamiento, destaca la alianza entre los burgueses, la Iglesia Católica y el Estado para implementar asistencia social (racional y científica) a través de la fundación de la Sociedad de Organización de la Caridad.

Ante ese escenario y en consecuencia con los planteamientos de Netto (1992), la mediación entre las condiciones desarrolladas da cabida a la génesis del Servicio Social como agente profesional que atiende a través de la política social la pauperización reproducida por la vivencia capitalista. Por lo tanto, en términos generales, es entendible la temprana presencia de la profesión en Europa respecto a América, suponiendo que en la parte occidental del viejo continente es donde inicialmente se expresa

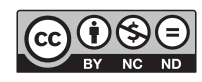

Rev. Reflexiones 98 (1) Enero-Junio, 2019: 23-37, E-ISSN: 1659-2859 
la maduración del capitalismo, comprendiendo este modelo de producción como el generador de los condicionantes para la génesis de la profesión.

Es pertinente señalar que el asentamiento de esas condiciones no implicó su inmediata constitución, pues tomó algo de tiempo contar con un perfil profesional relativamente amalgamado. La tendencia a nivel occidental fue que previo a la demanda y establecimiento de la educación formal en Servicio Social existieran por un intervalo agentes sociales interventores del conflicto social, nombrados por la literatura especializada como protoformas de la profesión.

Es posible ilustrarlos con el curso de visitadoras sociales ofrecido por la Sociedad de Organización de la Caridad en Inglaterra para el año 1893, mencionadas entre otros ejemplos por Martinelli (1989), la gama de oficios citados por Esquivel (2013) para efectos de Francia o bien, para el caso costarricense objeto de estudio en este documento: las visitadoras sociales de la década de 1930 mencionadas por Casas (1975) en su investigación. A pesar de ello, la tendencia muestra que una vez institucionalizada la formación universitaria en Servicio Social, estos agentes fueron sustituidos paulatinamente por los respectivos profesionales en la materia.

\section{Las principales tendencias formativas de la profesión y sus características religiosas en las emergentes Escuelas de Europa y los Estados Unidos a inicios del siglo $\mathrm{XX}$}

En ese sentido, la primera Escuela de Servicio Social en Europa fue fundada en Ámsterdam, Países Bajos, en el año 1899, Esquivel (2012) afirma que le fue seguida por Inglaterra en 1908, dos en París en 1911 y 1913, respectivamente, mientras que en año 1919 la Escuela de Filantropía Aplicada de Nueva York fue incorporada a la Universidad de Columbia. Al finalizar la Segunda Guerra Mundial se encontraban en funcionamiento cerca de 200 escuelas de Servicio Social distribuidas por Europa, Estados Unidos y América Latina; en esta región del continente americano la primera escuela se fundó en el año 1925, según indica Martinelli (1989).

El establecimiento de estas escuelas de Servicio Social debe ser entendido, según lo presentado, como la exigencia de las condiciones temporales y espaciales por contar con agentes de formación que intervinieran a través del respaldo institucional (Estatal o privado) en la atención del conflicto social ocasionado por el capitalismo. Por consiguiente, para efectos de este escrito es de interés mencionar las principales trayectorias de la profesionalización del Servicio Social, especialmente, destacando el carácter religioso que poseen y la exportación que de este hacen a otras latitudes del orbe, en miras a aportar al esclarecimiento de la influencia de la Iglesia Católica en la primera Escuela de Servicio Social en Costa Rica.

En la exposición de estas tendencias es posible identificar dos importantes epicentros originadores de trayectorias en Servicio Social, mismos que corresponden a las temporalidades y a las espacialidades antes presentadas, pero guardando entre sí notables diferencias; se habla de las tendencias producidas respectivamente en Europa Occidental y en la costa Este de los Estados Unidos durante el periodo finisecular del siglo XIX e inicios del siglo XX.

Afirma Martinelli (1989) que, en lo relacionado a este último país, a partir de 1897 es posible reconocer cursos dictados regularmente a personas voluntarias en la materia, los cuales tenían contenidos cercanos a la aplicación científica de la filantropía. La Escuela de Filantropía Aplicada de Nueva York, anteriormente mencionada, se deriva de ese proceso, constituyendo un importante soporte para la

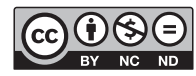

Rev. Reflexiones 98 (1) Enero-Junio, 2019: 23-37, E-ISSN: 1659-2859 
formación de lo que fue denominado en los Estados Unidos Social Work ${ }^{2}$, llegando al punto de sustituir la Sociedad de Organización de la Caridad para la década de 1940.

Mary Follet y Jane Adams apelaban a que la intervención debía orientarse a la armonización de las relaciones industriales y a la administración del conflicto social, mientras que Mary Richmond asociaba la práctica profesional con la asistencia social enfocada en la atención individual (Martinelli, 1989).

Las tendencias en las fundamentaciones empleadas en el Social Work estadounidense tenían como base la psicología, el psicoanálisis, la medicina y el derecho, además de la concepción de una actividad reformadora según plantea Martinelli (1989). En ese mismo movimiento, se podría suponer que el Social Work consideraba como referentes religiosos los principios evangélicos protestantes, generados por la susceptibilidad de estas denominaciones religiosas en los Estados Unidos, expresándose en tempranos momentos de la constitución profesional mediante el aparente discurso de la caridad, así como porque estos principios religiosos fueron coincidentes con los exportados desde Inglaterra en las Sociedades de Organizaciones de la Caridad. Sin embargo, frente a posturas religiosas Martinelli (1989) destaca más la tendencia a la búsqueda científica de la profesión concretado por ejemplo en las producciones de Mary Richmond.

Contrario a las tendencias del Social Work identificadas en los Estados Unidos, en Europa se desarrollaban vertientes en otra dirección, propias de las circunstancias que acompañaban la constitución profesional en esta espacialidad; más que un ajuste del individuo a su entorno, predominancia mostrada para el caso de los Estados Unidos, la preocupación de la tendencia profesional Europea fue la búsqueda por una explicación de lo sucedido a los sectores trabajadores, a través de fundamentaciones teóricas derivadas de autores como Frédéric Le Play, Auguste Comte y Émile Durkeheim, sociólogos conservadores en sus análisis de las relaciones sociales (Martinelli, 1989).

La doctrina social de la Iglesia Católica también coexistía como importante referencia en las fundamentaciones empleadas por el Servicio Social, dicha doctrina fue sustentada y desarrollada alrededor de las encíclicas papales Rerum Novaron del año 1891 del Papa León XIII y Cuadragésimo Anno del año 1931 del Papa Pío XI, según Martinelli (1989). Al respecto, Netto (1992) asegura que el catolicismo social se encontraba presente en las protoformas del Servicio Social, específicamente para el caso francés, pero sobre todo fue el centro de la configuración profesional en la región europea.

El vínculo del Servicio Social con la Iglesia Católica en Europa se evidenció más fuertemente en Bélgica, Italia, Portugal, España y Francia. En esta última nación, la primera Escuela de Servicio Social fundada para el año 1911 en París tuvo un importante papel en la difusión de esa referencia católica, al punto de hacer retroceder a la Sociedad de Organización de la Caridad que poseía un carácter evangélico. De las experiencias católicas impulsadas por esta instancia, surgieron espacios de organización, estudio e implementación en materia, conformándose como importante órgano para el año de 1925 en Italia, la Unión Católica Internacional de Servicio Social (UCISS), organización que tuvo peso no solamente en Europa, sino también en América Latina (Martinelli, 1989).

Alrededor de este asunto, Esquivel (2013) explicita las relaciones de la vertiente del Servicio Social francés en las primeras escuelas de América del Sur, aspectos que más adelante serán considerados en el análisis en curso. En lo que respecta a la vertiente religiosa desarrollada Netto (1992) asegura que las vinculaciones del Servicio Social europeo con la Iglesia Católica estuvieron presentes hasta aproximadamente la década de 1940.

En términos generales, a partir de los efectos de la Primera Guerra Mundial existió una recomposición en los principales centros del capital, Europa mostraba ya signos de descomposición en el liderazgo económico; la situación terminó de consolidarse posterior a la Segunda Guerra Mundial,

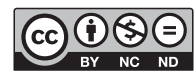

Rev. Reflexiones 98 (1) Enero-Junio, 2019: 23-37, E-ISSN: 1659-2859 
cuando la hegemonía ejercida por Europa fue obstaculizada por los estragos de dicho conflicto bélico, y subordinada por la ascendente economía y política estadounidense.

\section{Las expresiones de las tendencias de Europa y los Estados Unidos en la naciente formación profesional en América Latina durante la primera mitad del siglo XX}

En lo que respecta a la presencia de la profesión en América Latina, más específicamente su vinculación con la Iglesia Católica, resulta oportuno considerar lo que Esquivel (2013) denomina los tres polos geográficos y políticos que perfilan la base de germinación profesional en la región.

El autor en mención sitúa el primer foco en el cono sur de América Latina conformado por Chile, Brasil, Uruguay, Paraguay y Argentina, receptores de la herencia francesa con importantes implicancias de la Iglesia Católica; el segundo lo establece por países localizados entre Venezuela, Colombia y Ecuador mostrándose una vertiente europea y una incipiente orientación norteamericana ante el ascenso de esta última en el escenario internacional a partir de la primera mitad del siglo XX; el tercer grupo de países los ubica el autor en el istmo centroamericano, junto con Panamá, México y el Caribe, indicando la existencia de diferencias importantes entre las naciones de este último foco (Esquivel, 2013).

En ese sentido, partiendo del entramado expuesto, se procede a indagar las circunstancias iniciales de la primera Escuela de Servicio Social en Costa Rica aportando, específicamente, al debate de su relación con la Iglesia Católica.

\section{Condicionantes para la génesis y reproducción del Servicio Social en Costa Rica}

Plantea Esquivel (2007) que para identificar la génesis y reproducción del Servicio Social en Costa Rica es necesario considerar en tal motivación analítica, los insumos que coloca el capitalismo local, las manifestaciones de la cuestión social y el Estado Liberal, para concretarse posteriormente la génesis profesional, durante un periodo de cambios locales provocados por los movimientos del capitalismo internacional y acompañados por las configuraciones reformistas que se experimentaban en el país; planteamientos coincidentes con las propuestas de comprensión de la constitución profesional desarrolladas en este escrito.

Es posible identificar en Costa Rica durante el último cuarto del siglo XIX aspectos que vinculan al país con el capitalismo internacional, así como el consecuente establecimiento de un Estado que, motivado por esas dinámicas, asume rasgos liberales. Las investigaciones de Araya (1982) y Botey (2005), señalan que el café producido en la Meseta Central fue un importante dinamizador de la economía, así como las plantaciones de banano que surgen especialmente en la zona Atlántica, en paralelo a la construcción de la línea ferroviaria que transportaba el café al principal puerto de exportación del país, representaron también significativos productos en las relaciones económicas que se establecían en Costa Rica (Araya, 1982 y Botey, 2005). A partir del periodo en mención, se evidencia la hegemonía de sectores liberales en la conducción estatal, se realizaron importantes reformas en lo relacionado con la salud, la educación y la centralización de la creciente institucionalidad pública.

En correspondencia con las ideas liberales, se concretaron acciones por retirar a la Iglesia Católica del dominio institucional, expresado en las leyes anticlericales, según afirma Vargas (1989). Por su parte, Molina (2009) indica que el énfasis de la Iglesia Católica en lo espiritual entre 1880 a 1930 conlleva a que su quehacer no se asemeje a países como Brasil, Argentina, Chile y Uruguay donde si hubo una preocupación más sistemática y temprana por los sectores trabajadores.

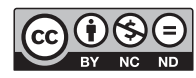

Rev. Reflexiones 98 (1) Enero-Junio, 2019: 23-37, E-ISSN: 1659-2859 
Durante el periodo transcurrido entre las dos guerras mundiales es posible identificar en Costa Rica la presencia del llamado Estado Reformista, el cual según rasgos indicados por Esquivel (2007) a través de una cita realizada en el texto de Salazar y Salazar (1992), tenía un carácter de desarrollo industrial, de intervención pública, de generación de asistencia social, de defensa de la educación y poseía planteamientos de defensa a la democracia liberal.

$\mathrm{Al}$ concretar los principales rasgos de ese periodo en diferentes ángulos de la sociedad, a nivel económico se evidencia una composición que continuaba siendo dinamizada por el café y el banano, lo cual mantenía la supeditación económica pendiente a los vaivenes de las crisis internacionales, igualmente colocado en esa dinámica de producción pero en segundo bloque de importancia económica, se encontraban las producciones de cacao, azúcar, minerales y ganadería (Araya, 1982; Botey, 2005 y Molina y Palmer, 2008).

Por su parte, las tensiones experimentadas entre la oligarquía costarricense y los sectores trabajadores ante ese orden que se asentaba, se traduce a nivel político en la organización de los segundos como medio para colocar en la órbita estatal sus demandas, alcanzando notables conquistas en esas confrontaciones, por citar algunos ejemplos se mencionan, la huelga por la jornada laboral de 8 horas en 1920, la conformación del Partido Reformista en 1923, el Partido Comunista en 1931, así como la huelga de 1934 (Acuña, 1984 y Botey, 2005). En lo que respeta a la institucionalización de ese conflicto en el marco de la tendencia reformista, es posible mencionar la Ley de Inquilinato de 1922, Ley de Banco Nacional de Seguros y la Ley de Accidentes del Trabajo, ambas de 1925, también el establecimiento de instituciones como el Patronato Nacional de la Infancia de 1930 y la Secretaría de Salud Pública y Protección Social de 1931, entre otras (Botey, 2005).

En correspondencia con el primer apartado del escrito, señala Esquivel (2007) que no se identifica la fundación de un centro de enseñanza superior en Servicio Social como aspecto central para la génesis de la profesión, pues en su lugar tiene fundamental preponderancia el resultado de determinadas condiciones históricas tales como las antes desarrolladas. Agrega el autor que resulta igualmente importante para una profesión, en específico para el Servicio Social, el reconocimiento y la legitimidad social, aspectos que le dan sustento para ser incorporada en la educación superior. Para efectos del análisis en curso, dichas condiciones logran expresarse con claridad en la década de 1940, permitiendo la fundación de la primera Escuela de Servicio Social en Costa Rica.

Las condiciones en las que se origina y reproduce el Servicio Social en Costa Rica vienen influenciadas por las bases históricas desarrolladas anteriormente, pero en sus primeros años de institucionalización se ve configurado además por una particular coyuntura nacional originada en la década de 1940 .

Durante ese periodo se evidencia que el partido político oficial liderado por Rafael Ángel Calderón Guardia (1940 - 1944) se alía inicialmente con la jerarquía de los sectores católicos, entre otros aspectos, por la amenaza comunista en las urnas, pero posteriormente también hubo una siguiente articulación con los sectores comunistas, generando una importante reforma social en el país, expresada en la fundación de la Universidad de Costa Rica, el Código de Trabajo y la Caja Costarricense del Seguro Social pero posibilitando al mismo tiempo con ese actuar, la presencia en la institucionalidad pública de la Iglesia Católica a través de la acciones como la derogación de las leyes anticlericales del siglo XIX (Molina, 2009 y Molina, 2010).

En el año 1944 hubo un cambio presidencial, más no en las tendencias que gobernaron en la administración anterior, esto en suma con otros aspectos, motivó a una fracción opositora en el año 1948 a la organización de un enfrentamiento que desencadenó una guerra civil, la cual perdieron los grupos que se

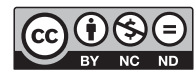

Rev. Reflexiones 98 (1) Enero-Junio, 2019: 23-37, E-ISSN: 1659-2859 
encontraban en ascenso y estaban relativamente aliados al inicio de la década de 1940 (Díaz, 2015). Por consiguiente, posterior a la guerra civil de 1948, hubo una reconfiguración de las fracciones políticas al mando de la conducción estatal, posibilitando que durante la segunda mitad del siglo XX, tuvieran cierta hegemonía los sectores vencedores del conflicto de 1948, quienes se adscribieron a la socialdemocracia a través del partido político denominado Liberación Nacional (Molina y Palmer, 2008).

\section{Condicionantes de la relación entre la Iglesia Católica y la Escuela de Servicio Social de la Universidad de Costa Rica}

Derivado del escenario en mención, la primera Escuela de Servicio Social en Costa Rica fue fundada en el año 1942, a través del impulso de un grupo de profesionales provenientes de diferentes disciplinas, quienes para la época participaban en el quehacer estatal. Según Mora (1969) la Escuela fue regida en sus inicios por un Consejo Directivo integrado por un director, sub-director, cuerpo docente, secretario y un delegado de cada una de las instituciones que subvencionaba al centro de estudios.

A partir del texto enviado por el abogado Héctor Beeche Luján al Secretario de Educación Pública Luis Demetrio Tinoco en el año 1942 solicitando y justificando la apertura de una Escuela de Servicio Social, es posible identificar nombres de personas que formaron parte de su primera estructura organizativa; en ese sentido, el Sr. Beeche Luján fungió como director, el Sr. Oscar Barahona Streber ocupó el rol de Sub-director y el Sr. Abel Guier Alvarado fue el secretario (Archivo Nacional de Costa Rica, 1942), además a partir de Mora (1969) es posible afirmar que entre las instituciones que financiaron dicha iniciativa académica, y por consiguiente participaron en tal Consejo Directivo, se encontraban el Patronato Nacional de la Infancia (PANI), la Caja Costarricense de Seguro Social (CCSS) y la Secretaría de Salubridad Pública.

A través de la misiva antes mencionada, enviada por el señor Beeche Luján (Archivo Nacional de Costa Rica, 1942), es posible reconocer que hubo un temprano interés por incorporar la Escuela a la recién fundada Universidad de Costa Rica, pero no fue posible su adscripción hasta el año 1944, momento en que el Consejo Universitario avaló su incorporación a la Universidad de Costa Rica (Consejo Universitario de la Universidad de Costa Rica, 1944).

El primer plan de estudios fue impulsado por la Association of School of Social Work, pero no logró concretarse, pareciera que tampoco hubo mayor recepción a la presentación realizada en materia por la Secretaría de la Unión Católica Internacional de Servicio Social (UCISS) durante el VIII Congreso Panamericano del Niño realizado en Costa Rica en el año 1939 (Esquivel, 2007; Angulo, 2014). La naturaleza de la profesión como síntesis de contradicciones políticas y económicas de la sociedad capitalista posibilita entender lo sucedido en relación con los actores sociales que demandaban la creación de la Unidad Académica, así como las fundamentaciones teórico-metodológicas que se expresaban en la Escuela.

A partir de la segunda década del siglo XX se desarrollaron una serie de reformas estatales para la contención de la creciente cuestión social, demandando la presencia de cuadros técnicos para las instituciones públicas. En un principio existió la figura de la visitadora social en programas como "La Gota de Leche" o en servicios sociales del PANI, pero posteriormente las labores desarrolladas por estos fueron subsumidas y sustituidas por profesionales de la Escuela de Servicio Social de la Universidad de Costa Rica.

Los fundamentos teórico-metodológicos de la formación durante el periodo de estudio fueron investigados por Fallas (2012), la autora afirma que estos se encontraban vinculados a las

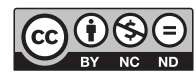

Rev. Reflexiones 98 (1) Enero-Junio, 2019: 23-37, E-ISSN: 1659-2859 
transformaciones del Estado, la política social y sus referentes ideológicos en su relación con la reproducción del capital, así como por los elementos filosóficos y teóricos predominantes durante la formación de profesionales. Al respecto centrándose en la primera mediación identificada por la autora, señala que el desarrollo histórico de Costa Rica permite identificar la vinculación de la formación profesional y la Escuela de Servicio Social con el Estado y los intereses de la fracción socialdemócrata, alineada a los interés de los países del capitalismo central, especialmente con los Estados Unidos, tal situación propició que con mayor acento a partir de 1950, los organismos internacionales influyeran en el desarrollo de la Escuela a través de un currículo asociado a las tendencias socialdemócratas (Fallas, 2012).

Derivado del escenario presentado, se plantea que para estudiar los condicionantes de la influencia de la Iglesia Católica en la Escuela de Servicio Social de la Universidad de Costa Rica son propuestos en este escrito dos elementos que permiten su compresión analítica. El primero de los elementos es la hegemonía en la región de los Estados Unidos que incide en una mayor predominancia del Social Work estadounidense en la formación profesional. El segundo elemento es la dificultad formativa y organizativa del clero para concretar un proyecto católico durante los primeros años de constitución de la Escuela de Servicio Social.

El primer elemento desarrollado es la hegemonía en la región de los Estados Unidos que incide en una mayor predominancia del Social Work estadounidense en la formación profesional, el cual no necesariamente es representante del ala católica de la profesión. En ese sentido, cabe mencionar que los dos primeros directores de la Escuela de Servicio Social entablaron sus contactos e intereses profesionales en los Estados Unidos. Héctor Beeche, director entre los años de 1942 a 1947, lo hizo después de una visita a este país abogando sin éxito a su regreso por el Servicio Social criminológico que allá conoció (Esquivel, 2007), mientras que Francisco Herrera que la dirigió entre los años de 1947 a 1972 aproximadamente, cursó un posgrado en Social Work paralelo a sus estudios en Ciencias Sociales realizados en los Estados Unidos durante los años de 1945 a 1947 (Valverde, 1992).

Las circunstancias alrededor de la formación de Francisco Herrera aportan para la comprensión de los elementos que condicionaron la influencia de la Iglesia Católica en la Escuela de Servicio Social; sin embargo, en lo relacionado con el primer elemento desarrollado se destaca que el sacerdote fue enviado a los Estados Unidos en el año de 1945 por el Arzobispo de la Arquidiócesis de San José, Víctor Manuel Sanabria. Los motivos del envío pueden explicarse según coloca Molina (2009), en el eventual interés de Sanabria por contar con sacerdotes formados en temas que serían importantes en el debate público ante la creciente política reformista.

Si bien lo anterior representa lo sucedido a nivel nacional, el ambiente en que fluctuó la formación de Francisco Herrera tenía otras características. A nivel internacional se evidenciaba el debacle de Europa no sólo en lo relacionado a la formación profesional, sino también a su papel en el escenario mundial respecto a la ascendente hegemonía de los Estados Unidos. Muestras de ello es como en una entrevista realizada a Francisco Herrera (Valverde, 1992), el sacerdote relataba que su interés inicial fue estudiar en Europa pero debido a la Segunda Guerra Mundial tuvo que postergar la decisión y cambiar el país en el que había planificado cursar sus estudios. En ese sentido, la tendencia predominante durante el periodo de formación de Herrera en los Estados Unidos, según lo desarrollado, aspiraba más por la profesionalización del Servicio Social (expresado en el Social Work) que, a uno de carácter religioso, y paciera que menos aún a uno católico.

$\mathrm{Al}$ respecto es señalado que, al asumir la conducción de la Escuela de Servicio Social, el sacerdote centró su labor en el establecimiento de una formación profesional más consistente frente a la que había encontrado a su llegada a la Universidad de Costa Rica. En la entrevista antes mencionada, así como otra realizada por el Eco Católico también en el año 1992, Herrera aseguró que cuando asumió

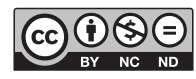

Rev. Reflexiones 98 (1) Enero-Junio, 2019: 23-37, E-ISSN: 1659-2859 
la dirección no habían títulos que acreditaran la formación profesional en Servicio Social (Valverde, 1992 y Quirós, 1992).

Cabe señalar que desde la década de 1940 se identifica la proximidad de organismos internacionales con la Escuela de Servicio Social pero su influencia se consolidó entre las décadas de 1950 a 1960 con la Organización de las Naciones Unidas (ONU) y la Organización de Estados Americanos (OEA) (Esquivel, 2007; Fallas, 2012 y Angulo, 2014). Una las principales cercanías entabladas durante el periodo en estudio, fue la injerencia de la ONU en el año 1953 quien sugirió modificaciones a los planes de estudios y organizó los centros donde se realizaban las prácticas profesionales (Fallas, 2012).

En ese sentido, las revisiones del primer plan de estudios de 1942, así como los de 1954 y 1965 periodo en que el sacerdote Francisco Herrera estuvo en la conducción de la Escuela, dan cuentas de la existencia de materias en la línea de las exigencias contextuales que perfilaban el desarrollo de la institucionalidad costarricense, más que la presencia específica de la influencia religiosa católica en la formación profesional.

A partir de la investigación de Campos, Molina, Molina, Romero y Ruíz (1977) es posible asegurar que el plan de 1942 poseía un marcado acento en la regulación de los individuos desde su ajuste con el medio, en el plan de 1954 no hay una distancia sustancial respecto a las fundamentaciones mencionadas pero existe una importante inflexión en el tanto son incorporados cursos prácticos a la formación, para el plan de 1965 persisten las fundamentaciones en cuestión pero las autoras destacan tres importantes características relacionadas con: metodologías de la práctica académica, búsqueda por la integración de unidades curriculares y la "autodiferenciación” profesional dentro de la práctica académica.

A través de las vinculaciones de los dos primeros directores con el Social Work, la temprana relación de la Escuela de Servicio Social con los organismos internacionales, así como las particularidades del país, es posible señalar la imposibilidad de la Unidad Académica para adscribirse a las tendencias internacionales de carácter católico, y por el contrario, su relación fue mayor con el Social Work estadounidense en suma con las demandas específicas de la sociedad costarricense.

El segundo elemento identificado es la dificultad formativa y organizativa del clero para concretar un proyecto católico durante los primeros años de constitución de la Escuela de Servicio Social.

Tal y como se ha indicado, en Costa Rica durante la década de 1940 se articuló una coyuntura que posibilitó el reposicionamiento de la Iglesia Católica como un actor político con injerencia estatal, respecto al limitado papel que había experimentado anteriormente en cuanto a la intervención en la cuestión social. Asegura Molina (2009) que los políticos católicos empezaron a comprender en la década de 1920 los réditos electorales que poseía incursionar en el campo social frente a centrar su programática política únicamente a la derogación de las leyes anticlericales, para el autor la derogación de esta normativa difícilmente sería considerada en el congreso si los temas sociales concentraban la atención, el peligro para el clero en este sentido se acrecentaba ante la canalización electoral de la desigualdad social por parte del naciente Partido Comunista en la década de 1930

En ese sentido, el prelado de San José para el periodo en estudio, Víctor Manuel Sanabria, al asumir la diócesis en el año 1940 afirmaba que se encontró con un clero poco sensibilizado con "las clases humildes", escaso y sin la formación para una serie de acciones que en la materia le interesaba emprender (Backer, 1978). Ante esos requerimientos y como fue mencionado anteriormente, una serie de sacerdotes fueron enviados al extranjero para realizar estudios superiores, entre los que destacan Benjamín Núñez y el antes mencionado Francisco Herrera. Ambos estudiaron en los Estados Unidos materias vinculadas con el área social, según se indica en el texto de Molina (2009), Núñez se

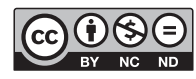

Rev. Reflexiones 98 (1) Enero-Junio, 2019: 23-37, E-ISSN: 1659-2859 
especializó en Ciencias Sociales, mientras que Herrera como fue señalado por Valverde (1992), estudió Ciencias Sociales y Servicio Social.

Ambos ocuparon puestos relacionados con sus formaciones. Durante la coyuntura de 1940 entre otros aspectos, el primero organizó un sindicato católico de trabajadores como medida para contrarrestar la organización comunista, el segundo fue director de la Escuela de Servicio Social por aproximadamente 25 años (Backer, 1978; Valverde, 1992).

Al parecer, el establecimiento de un proyecto político católico en Costa Rica estuvo relacionado con la coyuntura de la década de 1940 que favoreció las relaciones entre la Iglesia Católica y el gobierno central, específicamente, durante los años de 1940 a 1948, además dicho proyecto estuvo impulsado por Víctor Manuel Sanabria, al variar estos condicionantes, se dificultaba el asentamiento del proyecto católico en cuestión. Lo anterior posibilita entender por qué inicialmente los sacerdotes mencionados realizaron acciones próximas al quehacer estatal vinculado con la Iglesia Católica pero al cambiar las condiciones que potenciaban esa proximidad (la pérdida de la guerra civil en el año 1948 de los sectores políticos cercanos a la Iglesia Católica, así como la muerte de Víctor Manuel Sanabria en el año 1952), Francisco Herrera y Benjamín Núñez se aproximaron a la ascendente socialdemocracia, no necesariamente para impulsar un proyecto católico.

Durante dicha coyuntura de ascenso de la Iglesia Católica, en lo relacionado con la Escuela de Servicio Social de la Universidad de Costa Rica, algunos sectores del clero manifestaron el interés de potenciar a través de ella una tendencia formativa de carácter católico, pero en la dirección de lo planteado dicho proyecto no da cuentas de haberse concretado.

Un referente significativo que permite la reflexión para mostrar las evidencias en la línea de lo afirmado, es lo planteado por el corresponsal de Acción Católica en Washington Jimmy Fonseca al prelado costarricense Víctor Manuel Sanabria; Fonseca le mencionaba a Sanabria en marzo de 1942 que

Si viniera podríamos conseguir con facilidad algunas becas que he tratado de gestionar; el señor Beeche parece que ha fundado una Escuela de Servicio Social. Aquí la National sostiene la mejor quizá de los E.E.U.U. con dotación especial de becas; ya sabe, Monseñor lo beneficioso que sería para nosotros titular aquí a algunas de nuestras señoritas de Acción Católica (AHA, 1942; Caja 45, Folder E, oficio 70).

En la línea de la correspondencia sostenida con otros sectores religiosos, durante una conversación de Víctor Manuel Sanabria en el año 1947 con su homólogo venezolano Luca Guillermo Castillo a raíz de la fundación de una Escuela de Servicio Social Católica en ese país, Víctor Manuel Sanabria hizo una valoración sobre la Escuela costarricense:

Aquí existe una Escuela de Servicio Social, agregada a la Universidad Nacional, que, aunque no llena todas las condiciones que serían de desear desde el punto de vista católico positivo, puede considerarse como bien aceptable, pero naturalmente el ideal sería poder imitar el admirable ejemplo de V.E. y fundar una Escuela de Servicio Social netamente católica. Ojalá el señor nos conceda esa gracia tan singular (AHA, 1947. Caja 56, folder A, oficio 54).

Al respecto, unos meses después, en setiembre de 1947, la Unión Católica Internacional de Servicio Social (UCISS) le escribió a Víctor Manuel Sanabria para que les ayudara a extender sus vinculaciones en América Central y el Caribe, y de paso valorar la fundación de una Escuela de connotación

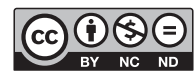

Rev. Reflexiones 98 (1) Enero-Junio, 2019: 23-37, E-ISSN: 1659-2859 
católica en Costa Rica (AHA, 1947, caja 56, Folder A, oficio 24). El secretario general de dicha organización, el señor Baers, le decía a Víctor Manuel Sanabria:

Creemos que en estos países [América Central y el Caribe] la Escuela Católica de Servicio Social responde mucho mejor a las necesidades de la población, y nos preguntamos si nos puede decir cómo llegar a promover escuelas católicas de servicio social. También nos gustaría saber si en su país una escuela de este tipo tendría lugar³ (AHA, 1947, caja 56, Folder A, oficio 24).

Las gestiones propuestas por la Unión Católica no eran casuales, para agosto de ese año, la Organización de las Naciones Unidas (ONU) había impulsado un congreso en Colombia en el cual le sugería a las autoridades de los países de América Central y el Caribe promover la fundación de Escuelas de Servicio Social. Pareciera ante ello, que la Unión Católica quería aprovechar la posible fundación de escuelas de Servicio Social para extender sus objetivos católicos a esta región de las Américas (AHA, 1947, caja 56, Folder A, oficio 24).

En cuanto a ello, la respuesta de Víctor Manuel Sanabria a la organización fue contraproducente respecto al lamento, anteriormente expresado a su homólogo venezolano por no contar en el país con una Escuela de connotación católica. Es planteado de esta manera, ya que, a pesar de agradecer el interés de la organización por gestar vínculos con el arzobispado costarricense, son más los temores que Sanabria expresa:

1) Existe ya una Escuela de Servicio Social, agregada a la Universidad de Costa Rica y mantenida por el Gobierno de la República, y difícilmente habría ambiente por el momento para otra Escuela semejante; 2) En esa Escuela son profesores dos sacerdotes que se han graduado en Ciencias Sociales en la Universidad de Washington, y mediante ellos se alcanza bastante en cuanto a la penetración de los ideales católicos entre los alumnos; 3) El sostenimiento de una Escuela de Servicio Social netamente nuestra, sin intervención del Estado, ofrecería además de la dificultad planteada antes, la de la imposibilidad de mantenerla económicamente (AHA, 1952. Caja 63. Folder J, oficio 47).

De esta manera, las palabras de Víctor Manuel Sanabria parecían centrarse más en su preocupación por las limitaciones económicas, que en el compromiso por llevar a cabo los propósitos de la Unión Católica; aunque, lo relacionado con los sacerdotes que daban clases en la Escuela resulta interesante para el análisis en curso.

No obstante, si se valora con detenimiento, realmente era poco lo que la Iglesia Católica pudo haber alcanzado a través de estos dos docentes para el momento de la carta, ya que Benjamín Núñez y Francisco Herrera llevaban tan solo un par de meses en la Escuela, y a lo sumo Núñez podía tener algún contacto no mayor a los dos años de antigüedad con la Escuela (Valverde, 1992); además, resulta inconsistente que en dicha misiva Víctor Manuel Sanabria no le mencionara a las autoridades de la Unión Católica que uno de los dos docentes era trabajador social y estaba a la conducción de la Unidad Académica.

A pesar de ello, ambas partes se comprometieron a gestar vínculos para que la unidad académica lograra formar parte de la Unión Católica. En cuanto a ello, cinco años más tarde, en un informe enviado por parte del arzobispado costarricense a la organización, los argumentos planteados no habían cambiado mucho respecto a la conversación sostenida en 1947.

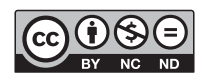

Rev. Reflexiones 98 (1) Enero-Junio, 2019: 23-37, E-ISSN: 1659-2859 
En cuanto a ello, nuevamente señalaron el argumento de los dos sacerdotes que ejercían la docencia, pero en esta ocasión hacían hincapié, a diferencia de la vez anterior, en que uno de ellos era director de la unidad académica; premisa que le dio cabida para afirmar que la Escuela tenía una connotación católica (AHA, 1952. Caja 63, folder J, oficio 47).

De igual forma, nuevamente señalan que era imposible formar un espacio académico privado debido a las serias dificultades económicas que atravesaba el país, además, "siendo la actual Escuela de Servicio Social, una institución de orientación católica, sería menos conveniente, no falsear aquella orientación, oponiéndole a la Escuela Universitaria, una escuela independiente católica" (AHA, 1952. Caja 63, folder J, oficio 47). En el mencionado informe, también señalaba que la dirigencia de la Central de Trabajadores Rerum Novarum se podría interesar en las propuestas de la organización, así como el sacerdote Francisco Herrera trabajaba en un plan para el desarrollo del Trabajo Social Católico.

De acuerdo con lo anterior, el informe en mención no es explícito sobre acciones concretas realizadas por los eclesiásticos para asumir las riendas de la Escuela de Servicio Social; incluso los argumentos que dan cabida para afirmar que ahora tienen una connotación católica no se distancia de los mencionados en 1947 cuando el discurso se centraba en las limitaciones para acoger las propuestas de la Unión Católica. Es decir, pareciera que se intentaba ver las mismas condiciones existentes en 1947 bajo una óptica más favorable.

El escaso trabajo que pudo haber realizado la Iglesia Católica para intervenir en la Escuela de Servicio Social, sin duda se pudo haber encontrado a mediados de 1952 el mismo destino que para esa fecha tuvo un eventual proyecto político de Víctor Manuel Sanabria. El 08 de junio de 1952 fallece el prelado, y su predecesor hizo poco en la línea de los doce años trabajados para el entonces ex arzobispo (Backer, 1978).

\section{Conclusiones}

A partir de lo presentado es posible comprender a la profesión como producto de las condiciones espaciales y temporales propias del asentamiento del capitalismo durante el periodo finisecular del siglo XIX e inicios del siglo XX, ocupando un papel creciente en la intervención estatal de la desigualdad generada por dicho modo de producción; hasta la institucionalización de la enseñanza profesional, mayoritariamente situada a inicios del siglo XX, existió un intervalo en que dicho abordaje fue desarrollado por variados agentes que ejecutaban prácticas cercanas al quehacer en materia.

En los dos epicentros donde inicialmente emergió la profesión, Europa y Estados Unidos, la contención, represión y control de las demandas presentadas por la población fue la tónica del abordaje; para tales fines y en correspondencia con los intereses del escrito, en la primera región hubo una cercanía mayor con la Iglesia Católica y en la segunda más bien hubo cercanía con preceptos evangélicoprotestantes, condicionados por la temprana búsqueda del carácter científico de la práctica profesional.

Mientras tanto, en América Latina la demanda e institucionalización de la enseñanza de la profesión se evidencia en la primera mitad del siglo XX, si bien las particularidades de cada nación del continente americano matizaron las características que poseía, pareciera que la hegemonía de Europa y los Estados Unidos en la región orientó en términos generales las matrices de ese emergente Servicio Social.

Para efectos de Costa Rica, la constitución del Servicio Social, especialmente de su formación profesional además de contener las particularidades históricas que le anteceden, es atravesada y configurada en sus primeros años por la coyuntura de la década de 1940, momento en que la Iglesia Católica

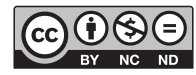

Rev. Reflexiones 98 (1) Enero-Junio, 2019: 23-37, E-ISSN: 1659-2859 
ocupó un papel político estratégico, principalmente en los primeros ocho años de dicha coyuntura. Durante ese periodo se intentan establecer relaciones entre la Iglesia Católica y la Escuela de Servicio Social de la Universidad de Costa Rica.

No obstante, a partir de lo desarrollado, se puede afirmar que una eventual experiencia formativa de Servicio Social Católico en la Universidad de Costa Rica debió afrontar una serie de situaciones de orden nacional e internacional que le imposibilitaron consolidarse.

A nivel internacional, existieron condiciones poco favorables para la adscripción a la corriente católica de carácter principalmente europeo, pues las vinculaciones formativas más se desarrollaron con los Estados Unidos. En el plano nacional, durante la década de 1940 la Iglesia Católica poseía un importante retraso entre otras cosas, en materia formativa producto del rezago y falta de organización que tuvo durante casi todo el siglo XIX, así como por las consecuencias de las leyes anticlericales, de igual forma, se encontraba sujeta a los vaivenes de esa coyuntura política que les reposicionó de manera temporal en la institucionalidad, mientras que la Escuela de Servicio Social poseía una marginal situación que le dificultaba establecerse como espacio formal a lo interno de la Universidad de Costa Rica.

Por lo tanto, hay evidencias para suponer que durante el periodo en estudio la Iglesia Católica costarricense pudo interesarse en impulsar a través de la Escuela de Servicio Social un proyecto formativo de carácter religioso pero las condiciones a nivel nacional e internacional imposibilitaron que dicha motivación se concretará formalmente en la Escuela de Servicio Social de la Universidad de Costa Rica.

\section{Notas}

1. Cabe aclarar que actualmente en Costa Rica la profesión en estudio es denominada como Trabajo Social, pero para efectos del presente escrito se mantuvo la designación de la época de Servicio Social.

2. Las personas integrantes de las Sociedades de Organización de Caridad norteamericanas defendían la denominación Social Work frente al Servicio Social (Social Service) aduciendo que caracterizaba con mayor precisión la dimensión de sus actividades (Martinelli, 1989).

3. Traducción propia, el documento original se encuentra redactado en francés.

\section{Referencias}

Acuña Ortega, Víctor Hugo. (1984). La huelga bananera de 1934. San José: Editorial CENAP-CEPAS.

Archivo Histórico Arquidiocesano. (1942). Fondos Antiguos. Sub-fondo Correspondencia Arzobispado Sanabria. Caja 45, Folder E, oficio 70.

Archivo Histórico Arquidiocesano. (1947). Fondos Antiguos. Sub-fondo Correspondencia Arzobispado Sanabria. Caja 56, folder A, oficio 54.

Archivo Histórico Arquidiocesano. (1947). Fondos Antiguos. Sub-fondo Correspondencia Arzobispado Sanabria. Caja 56, Folder A, oficio 24.

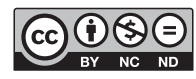


Archivo Histórico Arquidiocesano. (1952). Fondos Antiguos. Sub-fondo Correspondencia Arzobispado Sanabria. Caja 63. Folder J, oficio 47.

Archivo Nacional de Costa Rica. (1942). Fondo Gobernación, Sub-fondo Educación. Signatura 4722, folios. 60-78.

Angulo Brenes, Sonia. (2014). Fundamentos de la complejidad agraria-campesina y rural en la formación profesional de la Escuela de Servicio Social de la Universidad de Costa Rica, 1940 - 1970. (Tesis de maestría inédita). Universidad de Costa Rica, Costa Rica.

Araya Pochet, Carlos. (1982). Historia económica de Costa Rica 1821-1871. San José, Costa Rica: Editorial Fernández Arce.

Backer, James. (1978). La Iglesia y el sindicalismo en Costa Rica. San José: Editorial Costa Rica.

Botey Sobrado, Ana María. (2005). Costa Rica entre guerras: 1914-1940. San José, Costa Rica: Editorial Universidad de Costa Rica.

Campos Moreira, Ivette; Molina Cruz, Emilia; Molina Molina, Lorena; Romero Rodríguez, Carmen y Ruíz Rojas, Ana Isabel. (1977). Evolución de la estructura académica de la Escuela de Trabajo Social en Costa Rica. (Tesis de licenciatura inédita). Universidad de Costa Rica, Costa Rica.

Casas Fernández, Gerardo. (1975). Evolución histórica del Servicio Social costarricense. (Tesis de licenciatura inédita). Universidad de Costa Rica, Costa Rica.

Consejo Universitario de la Universidad de Costa Rica. (07 de marzo de 1944). Actas Históricas del Consejo Universitario. Acta 002. Recuperado de http://www.cu.ucr.ac.cr/uploads/tx_ucruniversitycouncildatabases/minute/1944/1944-002.pdf

Díaz Arias, David. (2015). Crisis social y memorias en lucha: guerra civil en Costa Rica, 1940 - 1948. San José: Editorial de la Universidad de Costa Rica.

Esquivel Corella, Freddy. (2007). Trabajo Social en Costa Rica: del ideario liberal a su constitución en el reformismo. San José: Editorial de la Universidad de Costa Rica.

Esquivel Corella, Freddy.(2012). Fundación de la primera unidad académica en el mundo: su contexto emergente en los Países Bajos. Revista Reflexiones, 91 (2), 151-162. Recuperado de https://revistas.ucr.ac.cr/index.php/reflexiones/article/view/1516/1524

Esquivel Corella, Freddy. (2013). Servicio Social francés: su impronta en la génesis del Trabajo Social de América del Sur. Revista Interacción y perspectivas, 3 (2), 122-146. Recuperado de http:// produccioncientificaluz.org/index.php/interaccion/article/view/508/507

Fallas Jiménez, Yessenia. (2010). Fundamentos teórico metodológicos del Trabajo Social costarricense, 1942/1970. (Tesis de maestría inédita). Universidad de Costa Rica, Costa Rica.

Fallas Jiménez, Yessenia. (2012). La cuestión teórico metodológica en el Trabajo Social costarricense: reflexiones desde su particularidad. Revista Reflexiones, 91 (2), 87-96. Recuperado de https:// revistas.ucr.ac.cr/index.php/reflexiones/article/view/1487/1496

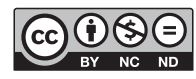


Hobsbawn, Erick. (2017). Historia del siglo XX. Barcelona: Editorial Planeta.

Martinelli, María Lúccia. (1989). Servicio Social: identidad y alienación. Sao Pablo: Cortez Editora.

Molina Jiménez, Iván y Palmer, Steven. (2008). Costa Rica del siglo XX al XXI. San José: Editorial Universidad Estatal a Distancia.

Molina Jiménez, Iván. (2009). Anticomunismo reformista, competencia electoral y cuestión social en Costa Rica (1931-1948). San José: Editorial Costa Rica.

Molina Jiménez, Iván. (2010). Los pasados de la memoria: El origen de la reforma social en Costa Rica (1938 - 1943). San José: Editorial Universidad Nacional.

Mora Rojas, Rosa María. (1969). Algunos aspectos relacionados con a formación de profesionales en la Escuela de Servicio Social de la Universidad de Costa Rica. (Tesis de licenciatura inédita). Universidad de Costa Rica, Costa Rica.

Netto, José Paulo. (1992). Capitalismo monopolista y Servicio Social. Sao Pablo: Cortez Editora.

Quirós, José. (10 de mayo, 1992). En el 50 aniversario de la fundación de la Escuela de Trabajo Social, el Pbro. Francisco Herrera Mora. Eco Católico, sin especificar número de página.

Valverde Obando, Luis. (1992). Los inicios del Trabajo Social en Costa Rica: El padre Herrera. Revista de Ciencias Sociales. (56), 43-50.

Vargas Arias, Claudio. (1989). El Liberalismo y la consolidación del Estado en Costa Rica. (Tesis de maestría inédita). Universidad de Costa Rica, Costa Rica. 
